\section{ORIGINAL RESEARCH}

J.Y. Jeon

I. Kovanlikaya

J.A. Boockvar

X. Mao

B. Shin

J.K. Burkhardt

K. Kesavabhotla

P. Christos

H. Riina

D.C. Shungu

A.J. Tsiouris

\title{
Metabolic Response of Glioblastoma to Superselective Intra-Arterial Cerebral Infusion of Bevacizumab: A Proton MR Spectroscopic Imaging Study
}

BACKGROUND AND PURPOSE: SIACI of bevacizumab has emerged as a promising novel therapy in the treatment of recurrent GB. This study assessed the potential of ${ }^{1} \mathrm{H}-\mathrm{MRS}$ as an adjunctive technique in detecting metabolic changes reflective of antiproliferative effects of targeted infusion of bevacizumab in the treatment of GB.

\begin{abstract}
MATERIALS AND METHODS: Eighteen patients enrolled in a phase I/II study of SIACl of bevacizumab for treatment of recurrent GB were included. Concurrent MR imaging and ${ }^{1} \mathrm{H}-\mathrm{MRS}$ scans were performed before and after treatment. Five distinct morphologic ROls were evaluated for structural and metabolic changes on MR imaging and ${ }^{1} \mathrm{H}-\mathrm{MRS}$, which included enhancing, nonenhancing $\mathrm{T} 2$ hyperintense signal abnormality, and multiple control regions. Pre- and post-SIACl of bevacizumab peak areas for NAA, $\mathrm{tCho,} \mathrm{tCr}$, as well as $\mathrm{tCho/tCr}$ and $\mathrm{tCho/NAA}$ ratios, were derived for all $5 \mathrm{RO}$ ls and compared using the Wilcoxon signed-rank test.
\end{abstract}

RESULTS: A significant median decrease of $25.99 \%$ (range -55.76 to $123.94 ; P=.006$ ) in tCho/NAA was found post-SIACl of bevacizumab relative to pretreatment values in regions of enhancing disease. A trend-level significant median decrease of $6.45 \%$ (range -23.71 to $37.67 ; P=.06$ ) was noted in tCho/NAA posttreatment in regions of nonenhancing T2-hyperintense signal abnormality.

CONCLUSIONS: The results of this ${ }^{1} \mathrm{H}-\mathrm{MRS}$ analysis suggest that GB treatment with $\mathrm{SIACl}$ of bevacizumab may be associated with a direct antiproliferative effect, as demonstrated by significant reductions of tCho/NAA after the intervention.

ABBREVIATIONS: $\mathrm{GB}=$ glioblastoma; $\mid \mathrm{A}=$ intra-arterial; $\mathrm{IDL}=$ Interactive Data Language; Lac = lactate; PFS = progression-free survival; RANO = response assessment in neuro-oncology; $\mathrm{SIACI}=$ superselective intra-arterial cerebral infusion; $\mathrm{tCho}=$ total choline; $\mathrm{tCr}=$ total creatine

G lioblastoma $(\mathrm{GB})$ is the most common malignant primary brain tumor. Despite an aggressive multimodal treatment approach that includes surgical resection and chemoradiation, median survival is limited to approximately 14 months. ${ }^{1}$ In May 2009, intravenous administration of bevacizumab (Avastin; Genentech/Roche, South San Francisco, California) gained FDA approval and emerged at the forefront as a treatment option in the setting of recurrent GB. ${ }^{2,3}$ Bevacizumab is

Received December 8, 2011; accepted after revision January 28, 2012.

From the Departments of Neuroradiology (J.Y.J., I.K., A.J.T.) and Neurosurgery (J.A.B., B.S., J.K.B., K.K.), New York Presbyterian Hospital, Weill Cornell Medical College, New York, New York; Citigroup Biomedical Imaging Center (X.M., D.C.S.) and Department of Biostatistics and Epidemiology (P.C.), Weill Cornell Medical College, New York, New York; and Department of Neurosurgery (H.R.), New York University Medical Center, New York, New York.

Dr. John Boockvar was supported by the National Cancer Institute Grant No. CA130985. Dr. Paul Christos was partially supported by the Clinical Translational Science Center Grant (UL1-RR024996).

Previously presented in part as a scientific oral exhibit at: Annual Meeting of the American Society of Neuroradiology; June 7, 2011; Seattle, Washington.

Please address correspondence to A.J. Tsiouris, MD, Associate Professor of Clinical Radiology, New York Presbyterian Hospital, Weill Cornell Medical College, Department of Radiology, 525 E 68th Street, New York, NY 10065; e-mail: apt9001@med. cornell.edu

Indicates open access to non-subscribers at www.ajnr.org

Indicates article with supplemental on-line table.

http://dx.doi.org/10.3174/ajnr.A3091 a monoclonal antibody that is believed to function primarily as an anti-angiogenic agent through inhibition of vascular endothelial growth factor. ${ }^{4}$ To overcome associated systemic side effects, toxicity, and impedance of the blood-brain barrier, a delivery method based on transient blood-brain barrier disruption with mannitol and known as SIACI of bevacizumab, was recently described. ${ }^{5,6}$ This novel delivery technique of dose augmentation has demonstrated safe and promising phase I results and may provide a more targeted treatment strategy. A recently completed phase II trial of SIACI of bevacizumab for GB treatment has revealed efficacious outcomes. $^{7}$

While treatment with bevacizumab produces dramatic decreases in MR imaging contrast enhancement, the degree to which this radiographic finding reflects an actual tumoricidal effect remains unclear. Although recognized to act as an antiangiogenic agent, its concurrent reduction of abnormal tumoral vessel permeability, marked by improved regional edema, may also contribute to changes in enhancement features. $^{8-11}$ Furthermore, the extent of a true cytotoxic antiglioma effect has yet to be elucidated at the molecular level. As a result, this complex interplay confounds the nonspecific relationship between enhancement and tumor burden; the extent to which changes in MR imaging enhancement features are attributable to anti-angiogenic effects, altered vessel characteristics, and/or tumor cell death remain to be established.

The inability of routine contrast-enhanced MR imaging to 


\begin{tabular}{|c|c|}
\hline Pulse Sequence & Scan Parameters \\
\hline \multicolumn{2}{|l|}{ T1WI 3-plane localizer } \\
\hline Sagittal T1WI, FSE & $\begin{array}{l}\text { TR/TE: } 600 \text { ms/minimum; FOV: } 250 \text { mm; } 352 \times \text { 256; ST/gap: 5/0 mm; NEX: 1; ETL: 2; full } \\
\text { echo }\end{array}$ \\
\hline Axial T1WI, FSE & TR/TE: 425/20 ms; FOV: 220 mm; $352 \times$ 224; ST/gap: 5 mm/0; NEX: 1; ETL: 2; full echo \\
\hline Axial T2WI FLAIR & TR/TE: 9600/140 ms; FOV: 220 mm; 352 × 224; ST/gap: 5 mm/0; NEX: 1; full echo \\
\hline Axial GRE & $\begin{array}{l}\text { TR/TE: } 635 \text { ms/minimum; FOV: } 220 \text { mm; } 320 \times \text { 224; ST/gap: } 5 \text { mm/0; NEX: 1; flip angle: } \\
\text { 20; full echo }\end{array}$ \\
\hline Axial DWI, EPI & $\begin{array}{l}\text { TR/TE: } 8200 \text { ms/minimum; FOV: } 220 \text { mm; } 128 \times \text { 128; ST/gap: } 5 \text { mm/0; NEX: 1; no. of } \\
\text { shots: 1; full echo }\end{array}$ \\
\hline Axial T2WI FSE & TR/TE: 3167/85 ms; FOV: 220 mm; 416 × 256; ST/gap: 5 mm/0; NEX: 2; ETL: 23 \\
\hline Axial T1WI FSE, postcontrast & TR/TE: 425/20 ms; FOV: 220 mm; $352 \times$ 224; ST/gap: 5 mm/0; NEX: 1; ETL: 2; full echo \\
\hline Sagittal 3D spoiled GRE (BRAVO), postcontrast & $\begin{array}{l}\text { TR/TE/TI: } 450 \text { ms/minimum/450 ms; FOV: } 307 \text { mm; } 256 \times 256 \times 136 \text {; ST: } 1.2 \text { mm; NEX: } \\
\text { 1; flip angle: } 15^{\circ}\end{array}$ \\
\hline Dynamic contrast-enhanced perfusion MRI, gradient-echo EPI & $\begin{array}{l}\text { TR/TE: } 2000 \text { ms/minimum; FOV: } 240 \text { mm; } 129 \times \text { 96; ST/gap: } 5 \text { mm/0; NEX: 1; no. of } \\
\text { shots: 1; flip angle: } 60^{\circ}\end{array}$ \\
\hline Axial multisection ${ }^{1} \mathrm{H}-\mathrm{MRS}$ & $\begin{array}{l}\text { TR/TE: 2300/280 ms; FOV: } 240 \text { mm; } 20 \times \text { 20; ST/gap: 15/3.5 mm; NEX: 1; } 4 \text { sections, } \\
\text { interleaved. }\end{array}$ \\
\hline
\end{tabular}

Note:-ETL indicates echo-train length; GRE, gradient-recalled echo; ST, section thickness.

differentiate between response, progression, and/or treatment effects has led to increased interest in evaluating ${ }^{1} \mathrm{H}-\mathrm{MRS}$ as a complement to contrast-enhanced MR imaging in the evaluation of intracranial lesions. ${ }^{12-15}$ By measuring metabolite levels, rather than anatomic structure, MRS may provide information on the metabolic status of tumors and complement information on structural and signal abnormalities derived by MR imaging. Several studies evaluating MRS accuracy have indicated that it may serve as a powerful tool in assessing treatment effectiveness. ${ }^{12,14,15}$ Specifically, MRS studies have consistently documented elevated tCho and decreased NAA over time in patients with unsuccessful therapy outcomes, suggesting the value of these metabolites and their ratios as biomarkers of therapeutic response.

In the present study, we sought to assess the utility of brain metabolites, including tCho, NAA, tCr, lactate, and their ratios, measured by MRS in enhancing and nonenhancing components of GB after SIACI of bevacizumab, as biomarkers of therapeutic response. As prior studies have demonstrated tCho to decrease and NAA to stabilize or partially recover in primary CNS neoplasms after successful treatment, ${ }^{16}$ suggesting a tumoricidal mechanism, we hypothesize that a therapeutic response to SIACI of bevacizumab would be associated with decreased tCho/NAA ratios. To our knowledge, the utility of MRS in recurrent GB status post bevacizumab treatment, and particularly in patients receiving novel dose intensification with SIACI of bevacizumab following transient blood-brain barrier disruption with mannitol, has not been investigated.

\section{Materials and Methods}

\section{Subjects}

Eighteen adult patients from a larger ongoing serial phase I/II study of SIACI of bevacizumab were retrospectively studied with approval of the institutional review board. ${ }^{5}$ All patients provided written informed consent for the phase I/II SIACI of bevacizumab trial. The On-line Table provides the patient demographics and characteristics, including age, sex, response/nonresponse status based on RANO criteria, ${ }^{17}$ prior IV bevacizumab exposure, prior steroid exposure, IA bevacizumab dose administration, and the number of days from
SIACI of bevacizumab until the posttreatment MR imaging/MRS examinations. Inclusion criteria required recurrent World Health Organization grade IV glioblastoma refractory to prior combined radiation treatment and chemotherapy with temozolamide, as well as preand posttreatment standardized gadolinium-enhanced concurrent brain MR imaging and MRS within the timeframe of 1-10 days before and 15-30 days after SIACI of bevacizumab therapy.

\section{Treatment Protocol}

The delivery technique entailed implementing SIACI of mannitol to facilitate transient blood-brain barrier disruption, followed by SIACI of bevacizumab within 3 days of baseline MR imaging. More specifically, $10 \mathrm{~mL}$ of $25 \% 1.4 \mathrm{~mol} / \mathrm{L}$ mannitol were infused through a microcatheter into the region of interest. Subsequently, the appropriate dose of bevacizumab was infused over 15 minutes. Given that the phase I trial aimed to determine the maximum tolerated dose of SIACI of bevacizumab with analysis of 10 escalating doses $(2,4,6,8$, $10,11,12,13,14$, and $15 \mathrm{mg} / \mathrm{kg}$ ), the administered dose varied among patients selected for our study. After 3-5 weeks of observation (range 15-30 days; mean 22 days), patients underwent follow-up brain MR imaging and MRS examinations. No additional therapy was initiated before the post-IA infusion MR imaging/MRS studies were completed.

Prior intravenous bevacizumab treatment was not an exclusion criterion for the phase I/II trials or for this study, with 6 patients having received previous IV bevacizumab exposure. For patients with more than 1 post-IA therapy MR imaging and MRS examination, only the initial post-IA infusion MR imaging and MRS studies were analyzed.

\section{Brain MR Imaging and MRS Data Acquisition and Processing}

All neuroimaging examinations were conducted on a 3T HDxt 15x MR system (GE Healthcare, Milwaukee, Wisconsin). The imaging protocol for patients with GB consisted of the standardized pre- and postcontrast imaging protocol provided in Table 1, and a multisection MRSI scan, which was performed as the final series in the study. The latter MRSI examination was performed using the multisection method of Duyn et al, ${ }^{18}$ which is a section-interleaved spin-echo sequence that incorporates octagonally tailored outer volume presatu- 


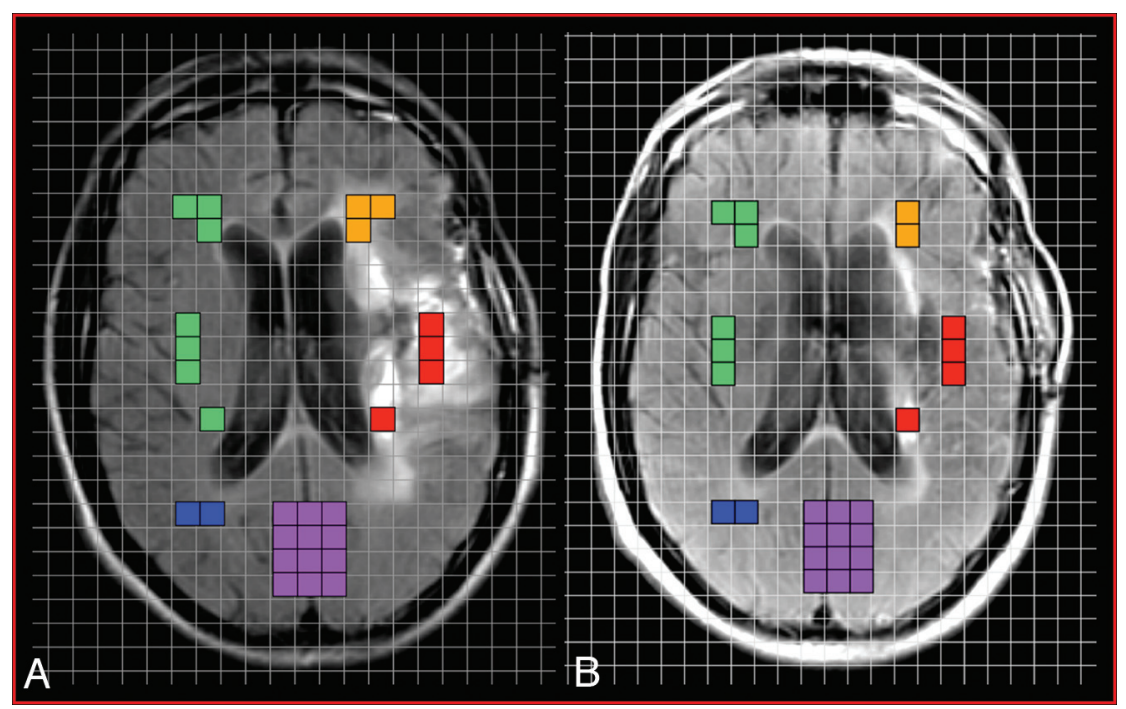

Fig 1. Representative example of voxel placement onto registered grid overlay on the pre-SIACI (A) and post-SIACI $(B)$ of bevacizumab T2-weighted FLAIR MRS localizer images demonstrating the 5 aforementioned regions of interest selected for analysis: red $=$ enhancing component orange $=$ nonenhancing T2-hyperintense signal abnormality; green = matched contralateral "normal" parenchyma (corresponding to cumulative area of enhancing and nonenhancing voxels); blue $=$ normal contralateral white matter; purple $=$ normal cortex. ROls were selected on the pretreatment T2-weighted FLAIR MRS $(A)$ and these 5 identical anatomic areas were then carried over and plotted on the posttreatment T2-weighted FLAIR MRS $(B)$.

ration pulses for pericranial fat and tissue suppression, and a single water-selective radio-frequency pulse followed by strong spoiler gradients for water suppression. The data were recorded in approximately 10 minutes from 4 interleaved $15-\mathrm{mm}$ brain sections-prescribed to encompass the tumor mass-with TE/TR 280/2300 ms, FOV $240 \mathrm{~mm}, 20 \times 20$ phase-encoding steps with circularly sampled $k$-space, 512 time-domain points, and $2500-\mathrm{Hz}$ spectral width. The resulting nominal MRS voxel size was $1.2 \times 1.2 \times 1.5 \mathrm{~cm}^{3}$.

The MRS data thus recorded were transferred to an off-line workstation (Sun Blade 1000; Sun Microsystems) for analysis by 2 blinded study investigators, who used proprietary data analysis software written in IDL (Exelis Visual Information Solutions, Boulder, Colorado). The raw data were sorted by section, zero-filled to a spatial matrix of $32 \times 32$ and twice along the acquisition domain (to 2048 sample points), filtered with a Gauss-Lorentz window and a Hamming window along the time and spatial domains, respectively, and then processed by standard 3D fast Fourier transformation to yield an array of $32 \times 32$ spectra. The spectral data were automatically corrected for susceptibility shifts due to slight variations in magnetic field strength across the brain.

Mean metabolite areas in each of the ROIs were obtained by using a frequency-domain nonlinear least-squares fitting routine developed in-house in IDL, and then expressed as rations relative to the root mean square of the background noise in each voxel or as ratios of a metabolite of interest to another (eg, tCho/NAA or tCho/tCr).

\section{Selection of Regions of Interest and Data Evaluation}

A senior board-certified neuroradiologist (A.J.T.; 9 years of experience), a trained senior neuroradiologist (I.K.; 21 years of subspecialty experience), and a junior neuroradiologist (J.Y.J.; neuroradiology fellow) selected the voxels of interest for MRS data analysis on the pretreatment MR imaging examination by using a registered grid overlay on the corresponding T2-weighted FLAIR localizer images. Voxel selection was assigned by consensus agreement by at least 2 of the 3 neuroradiologists, who were blinded to the clinical outcomes.

Five morphologic categories or ROIs, demonstrated pictorially in Fig 1, were selected for MRS analysis: 1) enhancing component, 2) nonenhancing T2-hyperintense signal abnormality, 3) matched contralateral "normal" parenchyma (corresponding to cumulative area of enhancing and nonenhancing voxels), 4) normal contralateral white matter, and 5) normal cortex. Matched contralateral paren- chyma was designated as the control region of interest. These colorcoded voxels were plotted onto the pretreatment MR spectroscopic image and selected for analysis by utilizing the standard T1-weighted precontrast, T1-weighted postcontrast, and T2-weighted FLAIR images from the baseline MR imaging performed the same day. The number of voxels per region of interest was variable, depending on the imaging characteristics of each neoplasm.

The corresponding anatomic areas for the same 5 morphologic categories were then carried over to the posttreatment MR imaging examination and similarly plotted onto a registered grid overlay on the T2-weighted FLAIR MRS localizer images. In cases where regional anatomy became altered after treatment, the focus remained on verifying that the same anatomic area was selected on the postinfusion study and reassigned as the correct region of interest for MRS analysis. In addition, in cases of discrepant section selection resulting in anatomic mismatch between the 4 pre- and posttreatment MRS T2weighted FLAIR localizer images, voxel placement was only performed on sections obtained at comparable anatomic section planes. Voxel selection was again verified by consensus agreement by at least 2 of the 3 study investigators.

In patients with enhancing and nonenhancing components of GB extending across the midline, the best collective judgment was made with regard to selection of matched "normal" parenchyma voxels, given the inability to select the corresponding contralateral cumulative area of enhancing and nonenhancing ROIs.

Upon completion of voxel placement for the 5 morphologic categories onto both the pre- and post-IA registered grid overlay on the T2-weighted FLAIR MRS localizer images, spectra were extracted from the corresponding MR spectroscopy voxels and then analyzed to assess for changes of tCho, tCr, Lac, and NAA levels and their ratios, as potential objective markers of therapeutic response.

\section{Data and Statistical Analysis}

Peak areas for 4 key metabolites (tCho, tCr, Lac, NAA) and their ratios ( $\mathrm{tCho} / \mathrm{Cr}$ and $\mathrm{tCho} / \mathrm{NAA}$ ) were derived for each of the 5 morphologic categories. Statistically significant differences in mean metabolite peak areas and ratios between the pre- and post-IA treatments (defined as percent change: [(postpre)/pre $\times 100 \%])$ were determined utilizing the Wilcoxon signed-rank test. Percent change for each MRS outcome was also compared between the ROIs of interest by the Wilcoxon signed-rank test. All analyses were performed using SPSS Ver- 


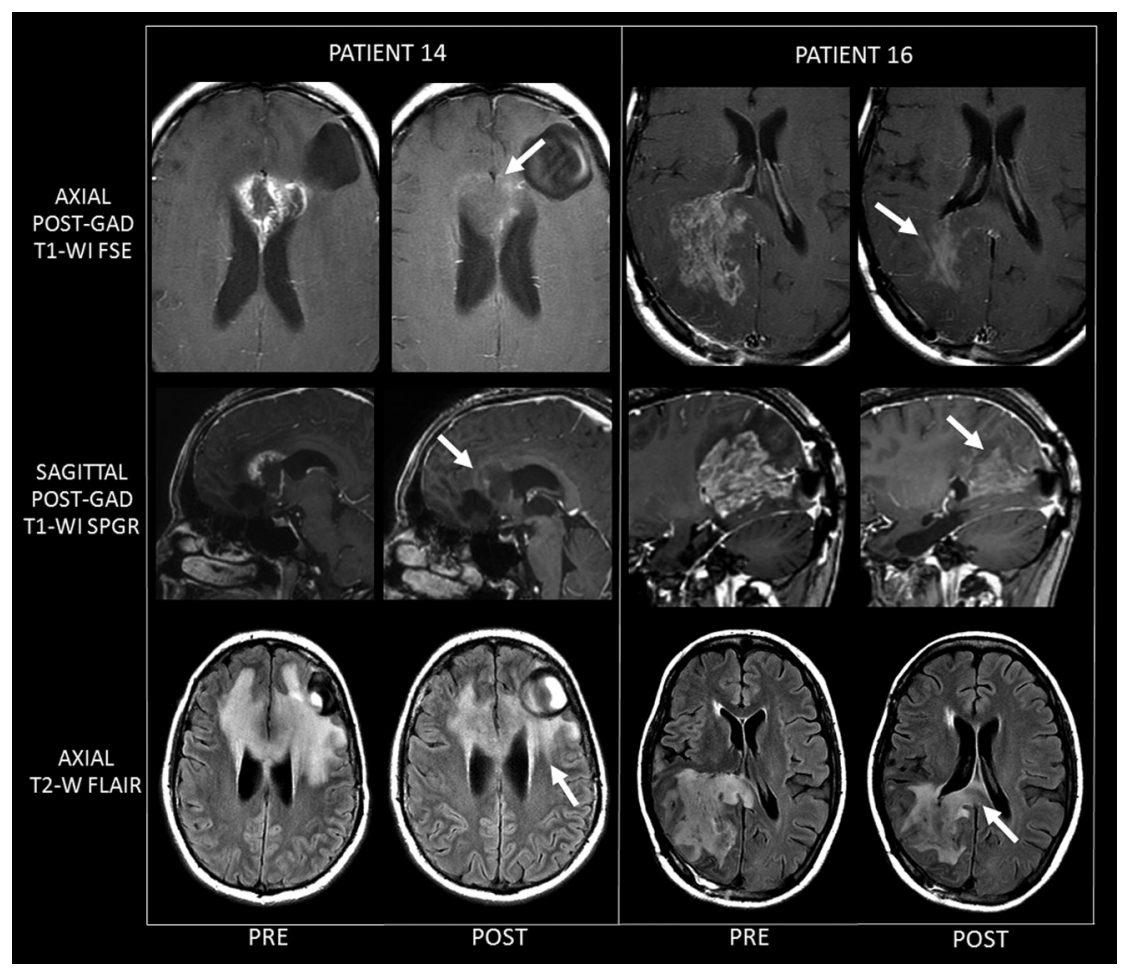

Fig 2. Two examples (study patients 14 and 16) of MR imaging changes before and after treatment, clearly demonstrating the marked decrease in enhancement associated with a mild decrease in T2 signal abnormality related to bevacizumab therapy. For each patient, images on the left are representative of pre-SIACI bevacizumab scans, and images on the right are matched representative post-SIACI bevacizumab scans. The arrows on the postgadolinium posttreatment T1WI point to the marked decrease in enhancement after SIACI of bevacizumab. Arrows on the posttherapy T2WI FLAIR images demonstrate a significant but less dramatic decrease in abnormal T2 signal. Patient 14 received 15 $\mathrm{mg} / \mathrm{kg}$ of SIACI of bevacizumab (1/2 left MCA, $1 / 4$ right $\mathrm{A} 1$ ACA, $1 / 4$ left A1 ACA). Patient 16 similarly received $15 \mathrm{mg} / \mathrm{kg}$ of $\mathrm{SIACl}$ of bevacizumab $(1 / 2$ right $\mathrm{P} 1 \mathrm{PCA}$ and $1 / 2$ right $\mathrm{M} 1$ $\mathrm{MCA})$

sion 19.0 (SPSS, Chicago, Illinois) and SAS Version 9.2 (SAS Institute, Cary, North Carolina) software by a trained biostatistician (P.C.). All reported $P$ values are 2 -sided, with statistical significance evaluated at $\alpha=.05$ level.

\section{Results}

Sample MR imaging results of changes in GB before and after SIACI of bevacizumab treatment, are demonstrated in Fig 2. These 2 examples (study patients $14 \& 16$ ) clearly demonstrate the marked decrease in enhancement associated with a mild decrease in T2 signal abnormality related to bevacizumab therapy.

Similarly, sample MRS data for a voxel in a GB in the splenium of the corpus callosum before and after SIACI of bevacizumab are shown in Figs $3 A,-B$, respectively. Pre-IA treatment GB spectra (Fig. $3 A$ ) were characterized by robust tCho and Lac increases, and decreased or absent $\mathrm{tCr}$ and NAA, with a decrease in overall spectral intensity. By contrast, spectral response after IA treatment (Fig 3B) generally consisted of recovery of the signal intensities of all the metabolites, with more marked increases in NAA and tCr levels and more modest changes in tCho and Lac levels.

The results of comparing median percent changes in $\mathrm{tCho/}$ NAA, our primary outcome measure, for each of the 5 ROIs, are provided in Table 2. The individual median percent changes are the medians computed from multiple sections in each subject. The values in the last row of Table 2 are the medians for all subjects. Comparing pre-IA versus post-IA treatment, there was a statistically significant median percent change of $-25.99 \%$ (range -55.76 to $123.94 ; P=.006$ ) in tCho/NAA in areas of enhancing disease, with a trend toward a significant change in this ratio (median percent change $-6.45 \%$; range -23.71 to $37.67 ; P=.06$ ) in areas of nonenhancing T2-hyperintense signal abnormality. The median percent change of the tCho/NAA ratio was not found to be statistically significant in the control and white matter regions
$(+7.04 \%, P=.12$, and $-3.67 \%, P=.80$, respectively), however it was statistically significant in cortical gray matter $(+8.62 \%, P=.02)$.

Median percent change in $\mathrm{tCho} / \mathrm{Cr}$ and tCho/NAA ratios were also compared between the 5 different brain region of interest groups. Median percent change in $\mathrm{tCho/Cr}$ ratios in all 18 subjects did not differ between the 5 region of interest groups, whereas statistically significant differences were found (Table 3) between the T2-hyperintense enhancing category and the "matched control" $(P=.005)$, cortical gray matter $(P=.004)$, and white matter $(P=.006)$ regions. Similarly, statistically significant differences in median percent change were found in the tCho/NAA ratios between the T2-hyperintense nonenhancing and gray matter regions $(P=.008)$, between the enhancing and T2-hyperintense nonenhancing regions $(P=.03)$, and between the white and gray matter regions $(P=.039)$. A trend toward significant differences was found in median percent changes in the tCho/NAA ratios between the T2hyperintense nonenhancing category and the "matched control" $(P=.11)$ and white matter $(P=.058)$ regions. No statistically significant differences were found between the control and gray matter, and control and white matter areas.

\section{Discussion}

The inability of conventional MR imaging to permit reliable clinical assessment of tumor progression and therapeutic response has heightened the need for the development of more effective neuroimaging approaches. In this respect, the emergence of alternate modalities has expanded the role of imaging beyond anatomic structural information to enable the analysis of physiologic and metabolic processes that may underlie neoplastic transformation. ${ }^{12,14,19-22}$ Particularly noteworthy is the utility of MRS in complementing conventional MR imaging for assessing treatment efficacy ${ }^{12,14}$; MRS is especially attractive in the setting of recurrent $\mathrm{GB}$, given the association of 
Pre-SIACI of bevacizumab
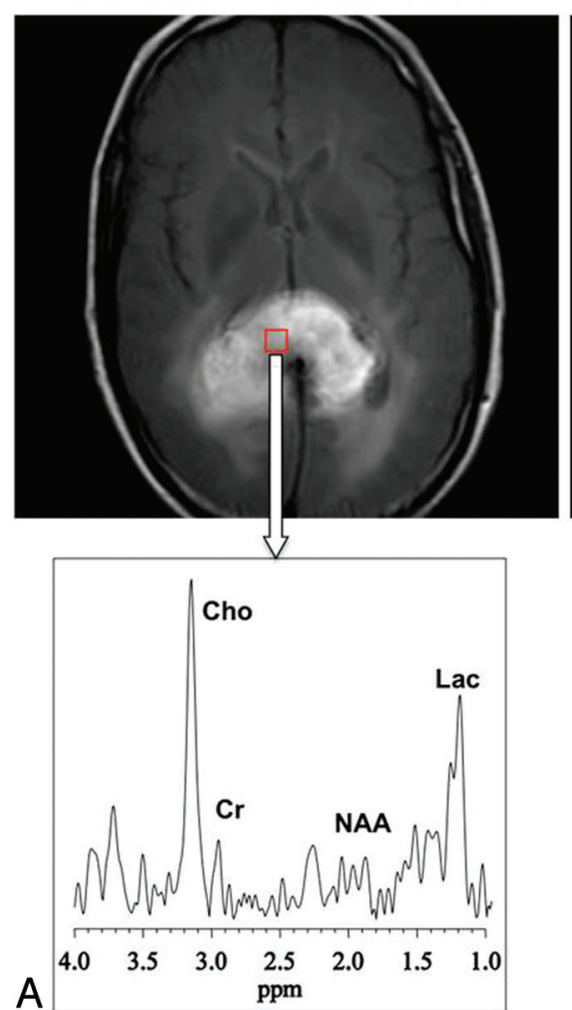

Post-SIACl of bevacizumab
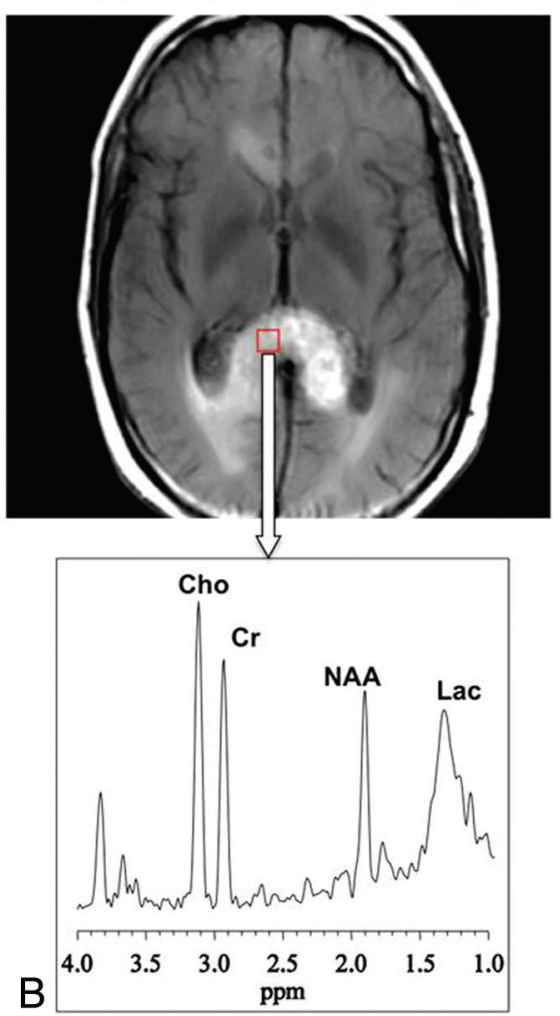

Fig 3. ${ }^{1} \mathrm{H}-\mathrm{MR}$ spectra for a voxel in a GB in the splenium of the corpus callosum before $(A)$ and after $(B)$ SIACI of bevacizumab, demonstrating reduction of the tCho/NAA ratio posttreatment.

\begin{tabular}{|c|c|c|c|c|c|c|}
\hline Patient & $\begin{array}{c}\text { Median \% Change } \\
\text { tCho/NAA } \\
\text { Enhancing }\end{array}$ & $\begin{array}{c}\text { Median \% Change } \\
\text { tCho/NAA } \\
\text { Nonenhancing }\end{array}$ & $\begin{array}{c}\text { Median \% Change } \\
\text { tCho/NAA } \\
\text { Control }\end{array}$ & $\begin{array}{c}\text { Median \% Change } \\
\text { tCho/NAA White } \\
\text { Matter }\end{array}$ & $\begin{array}{c}\text { Median \% Change } \\
\text { tCho/NAA Gray } \\
\text { Matter }\end{array}$ & $\begin{array}{c}\text { RANO } \\
\text { Response } \\
\text { Assessment }^{26}\end{array}$ \\
\hline 1 & 123.94 & 4.05 & 24.95 & -12.89 & -3.57 & SD \\
\hline 2 & -24.83 & -8.94 & 33.59 & -4.44 & 13.08 & $\mathrm{SD} / \mathrm{PD}^{*}$ \\
\hline 3 & 5.35 & -3.47 & 8.38 & 9.93 & -4.51 & SD \\
\hline 4 & -27.15 & -23.71 & 5.72 & 2.43 & 6.21 & SD \\
\hline 5 & -19.18 & 37.67 & 3.28 & -12.81 & 11.65 & SD \\
\hline 6 & -7.72 & -22.19 & 10.08 & -19.42 & 10.04 & PD \\
\hline 7 & -28.17 & -16.29 & 14.55 & 1.95 & 9.17 & SD \\
\hline 8 & -32.11 & -15.96 & 4.68 & 0.64 & -3.43 & SD \\
\hline 9 & -37.56 & -3.74 & 6.12 & 21.37 & 22.80 & SD \\
\hline 10 & -13.95 & -4.12 & 17.50 & 17.75 & 8.96 & $P R$ \\
\hline 11 & -4.43 & -7.94 & 15.56 & -4.31 & 8.28 & SD \\
\hline 12 & -45.23 & -21.59 & 7.96 & -14.76 & -1.37 & SD \\
\hline 13 & -47.67 & -4.96 & 15.79 & -6.40 & 10.69 & SD \\
\hline 14 & -46.51 & -13.88 & -4.55 & -8.88 & 12.89 & PR \\
\hline 15 & 8.25 & 3.75 & -0.28 & 13.17 & -1.10 & SD \\
\hline 16 & -24.17 & 7.50 & -23.48 & -6.78 & 5.19 & PR \\
\hline 17 & -55.76 & 9.58 & -21.53 & 23.58 & 100.96 & PR \\
\hline 18 & -33.70 & -19.41 & -24.89 & -3.03 & -14.85 & PR \\
\hline Median $\%$ change of medians & $-25.99 \%$ & $-6.45 \%$ & $7.04 \%$ & $-3.67 \%$ & $8.62 \%$ & Summary \\
\hline (interquartile range) & $(-39.48$ to -6.90$)$ & $(-17.07$ to 3.83$)$ & $(-1.35$ to 15.62$)$ & $(-9.86$ to 10.74$)$ & $(-1.89$ to 11.96$)$ & $\mathrm{SD}=11$ \\
\hline & $(-55.76$ to 123.94$)$ & $(-23.71$ to 37.67$)$ & $(-24.89$ to 33.59$)$ & (-19.42 to 23.58$)$ & $(-14.85$ to 100.96$)$ & $P R=5$ \\
\hline $\begin{array}{l}P \text { value for median } \% \text { change } \\
\text { (vs } 0 \% \text { ) }\end{array}$ & $P=.006$ & $P=.06$ & $P=.12$ & $P=.80$ & $P=.02$ & $\begin{array}{r}P D=1 \\
S D / P D=1\end{array}$ \\
\hline
\end{tabular}

Note:- Listed values are compared to $0 \%$ change (null value) for each of the 5 parameters. RANO response assessment key: PR indicates partial response: $\mathrm{SD}$, stable disease; PD, progression of disease. For patient $2\left(^{*}\right)$, the targeted treated primary tumor was classified as SD; however, new discontinuous leptomeningeal enhancement designated the overall response progressio

bevacizumab with dramatically reduced nonspecific contrast enhancement. By using MRS to assess neurochemical changes that may be associated with bevacizumab, we hoped to eluci- date some aspects of the complex mechanisms that seem to underlie the tumoricidal effects of the intervention. These mechanisms may include inhibition of neovascularization 


\begin{tabular}{lcccccccccc}
\hline Table 3: Comparison of median percent change in tCho/NAA ratios between the $\mathbf{5}$ different ROI groups \\
\hline $\begin{array}{c}\text { Enhance } \\
\text { vs. Control }\end{array}$ & $\begin{array}{c}\text { Enhance vs. } \\
\text { Nonenhance }\end{array}$ & $\begin{array}{c}\text { Enhance } \\
\text { vs. Gray }\end{array}$ & $\begin{array}{c}\text { Enhance } \\
\text { vs. White }\end{array}$ & $\begin{array}{c}\text { Nonenhance } \\
\text { vs. Control }\end{array}$ & $\begin{array}{c}\text { Nonenhance } \\
\text { vs. Gray }\end{array}$ & $\begin{array}{c}\text { Nonenhance } \\
\text { vs. White }\end{array}$ & $\begin{array}{c}\text { Control } \\
\text { vs. Gray }\end{array}$ & $\begin{array}{c}\text { Control } \\
\text { vs. White }\end{array}$ & $\begin{array}{c}\text { White } \\
\text { vs. Gray }\end{array}$ \\
\hline$P$ value & .005 & .03 & .004 & .006 & .11 & .008 & .058 & .91 & .23 & .039 \\
\hline
\end{tabular}

Note:-The median percent change values (of all 18 patients' individual median percent change values) are compared between the 5 different brain categories.

and/or normalization of existing tumor vessels resulting in decreased peritumoral edema. ${ }^{4,8-11}$

Bevacizumab and other anti-angiogenic agents may result in marked nontumoral decreases in contrast enhancement, termed "pseudoresponse." This phenomenon is characterized by a posttreatment decrease in the enhancing portion of the lesion, with progression of the nonenhancing portion on T2weighted sequences, and may be observed as early as 1-2 days after initiation of therapy. ${ }^{17}$ Awareness and recognition of this apparent, favorable radiographic pattern of change is critical for appropriately interpreting treatment response change, as the striking reduction of contrast enhancement may partly reflect normalization of the blood-brain barrier and decreased tumoral vessel permeability, rather than a true decrease in tumor volume. ${ }^{17,23}$ These anti-angiogenic agents demonstrate marked reductions in contrast enhancement, with high response rates and 6-month progression-free survival, but have rather modest or no overall survival benefits. ${ }^{17,23}$ Currently, no particular imaging technique is capable of differentiating between a true response and pseudoresponse. ${ }^{24}$

Standardized criteria for assessment of treatment response have been published and include the Macdonald criteria from $1990^{25}$ and the updated proposed recommendations by the RANO Working Group in 2010. ${ }^{17}$ Although the initial Macdonald criteria were based upon CT, these have evolved with application to conventional MR imaging, which has become the standard noninvasive neuroimaging technique for assessment of tumor burden. According to this method that has served as the current benchmark for tumor assessment, evaluation of therapy response is based on the change in contrastenhancing lesion size via $2 \mathrm{D}$ perpendicular measurements of the enhancing region. Clinical factors also taken into consideration include steroid dosage and the neurologic state of the patient. Various important limitations of the Macdonald criteria have since been acknowledged, primarily the nonspecific nature of contrast enhancement reflecting breakdown of the blood-brain barrier resulting from various etiologies; not necessarily representing the equivalent surrogate of tumor burden; and the lack of assessment of the nonenhancing disease component, often representing a combination of nonenhancing neoplasm, edema, and the sequelae of treatment. Recognition of these shortcomings has resulted in updated criteria by the RANO group, which address the nonenhancing areas of T2 hyperintense signal abnormality. ${ }^{17}$

In Table 2, we detail the individual response rates for our cohort of 18 patients utilizing the updated RANO response criteria. While there are no comparable studies evaluating early radiographic response utilizing the updated RANO criteria for recurrent $\mathrm{GB}$, the best available data show that median PFS is 4.2 months for IV bevacizumab alone and 5.6 months for IV bevacizumab plus irinotecan. ${ }^{26}$ Recent data from an internal study at our institution that is in press and includes this phase I patient cohort have demonstrated more encouraging outcomes by using the RANO criteria, with median PFS increasing to approximately 10 months for patients treated with a single IA bevacizumab dose followed by IV bevacizumab delivery. ${ }^{7}$

Still, the inherent inability of routine contrast-enhanced MR imaging to differentiate between response, progression, and/or treatment effects has led to increased interest in metabolic and functional neuroimaging techniques based on MR and PET. ${ }^{27}$ Sophisticated MR methods now rely upon the hemodynamic properties of gliomas, which include cerebral blood volume, cerebral blood flow, vascular permeability, and blood vessel diameter. ${ }^{27}$ New PET radiotracers target neoplastic biologic activity, enabling more specific interrogation of tumor physiology with assessment of hypoxia and proliferation rate. $^{28}$

Proton MRS is an alternative surrogate marker that can assist in monitoring treatment response and in clinical decision making. By allowing direct neurochemical analysis of several potential biomarkers of disease, MRS may serve as an important adjunctive technique. ${ }^{12-15}$ Metabolites of potential clinical interest include the putative neuronal density and viability marker NAA, which is decreased in most brain lesions; the cell bioenergetics marker $\mathrm{tCr}$, whose resonance includes a contribution from phosphocreatine and remains unchanged under most in vivo conditions, aside from extensive tissue damage or disrupted enzymatic homeostasis; the cell membrane biosynthesis and metabolism marker tCho, which increases in cell membrane breakdown and proliferation; and the anaerobic energy metabolism marker Lac, which is increased with anaerobic glycolysis activity. ${ }^{12,21}$ Thus, MRS represents a viable noninvasive neuroimaging technique for probing the metabolic disturbances that may elucidate the mechanisms of action of this promising intervention.

Our use of MRS in the present study has demonstrated the potential of the technique, revealing significant and trendlevel significant reductions of tCho/NAA ratios in enhancing and nonenhancing regions of disease, respectively. Measurement of changes in tCho has emerged a reliable predictor and indicator of neoplastic response, ${ }^{12}$ in concordance with the results of the present study. Our failure to find statistically meaningful changes for the matched contralateral normal parenchyma, as well as for normal white matter control voxels, seems to add face validity to our statistically significant findings in the enhancing and nonenhancing components of disease. While the absence of a significant difference in the matched "normal" and white matter areas was expected, our finding of a significant increase in tCho/NAA ratios within the gray matter ROIs $(+8.62 \% ; P=.02)$ was unexpected. One consideration is that this finding may reflect treatment-related neuronal loss and gliosis occurring to a greater extent compared with treatment effects on the neoplasm. More specifically, this might be seen in the setting of NAA values decreasing to a greater extent relative to decreasing tCho values, which 
would result in an overall increase in the tCho/NAA ratio. In addition, the statistically significant differences in median tCho/NAA ratios between the enhancing ROIs compared with the control, gray matter, and white matter groups further suggest that GB treatment with SIACI of bevacizumab may be associated with an antiproliferative effect.

Discordant findings were noted in 2 patients, with unexpected increases in the tCho/NAA ratio from the pretreatment to posttreatment scans. Patient 1 demonstrated a $123.94 \%$ increase in tCho/NAA in the enhancing ROIs, and patient 5 showed a $37.67 \%$ increase in tCho/NAA in the nonenhancing T2 hyperintense voxels (Table 2 ). Possible contributing factors for these 2 outlier nonresponders include low bevacizumab dosing (both were early patients at the start of the dose escalation Phase I trial), differences in specific tumoral genetics of these patients' neoplasms, and/or prior exposure to IV bevacizumab (in the case of patient 5 ).

This study has a number of limitations that suggest caution in generalizing the results. First, the sample size of 18 subjects is relatively small, so this report should be viewed as an initial description and interim analysis of our clinical experience to date, which is nevertheless promising. Second, as 1 effect of bevacizumab is to decrease edema by reducing the abnormal permeability of tumor vessels, our observed decrease in tCho/ NAA ratios may, in part, reflect the replacement of normal brain tissue into the relevant voxel. Third, clinical heterogeneity among patients selected for inclusion into the phase I/II SIACI of bevacizumab trials warrants consideration. This included variation in prior IV bevacizumab status with 6 of the 18 patients having previous exposure. Ideally, only bevacizumab-treatment-naïve patients should have been included to allow more accurate assessment of the true implications of this potential therapy. Our group is currently seeking funding for a larger controlled prospective study to directly compare IA and IV therapy in bevacizumab-naïve patients with recurrent GB. Similarly, the study sample was heterogeneous with respect to previous steroid exposure, with 5 of the 18 patients being steroid naïve. These factors are potential confounders to clinical outcomes and study results. Furthermore, the number of days from SIACI treatment to the postinfusion MRS study was not uniform, ranging from 15-30 days.

Various technical limitations should also be considered. The subjective component of selecting matching ROIs on the pre- and posttreatment scans, despite interval morphologic changes, introduces sampling error. Thus to some extent, differences in tissue type sampling may partially contribute to the apparent changes in metabolite concentrations. Second, inherent differences in anatomic resolution and difficulty in precisely coregistering the MRS localizer voxels with the corresponding anatomic ROIs-due to differences in spatial scan parameters and resolution (eg, section thickness, gap) - further complicates analysis, both in terms of in-plane resolution and along the $\mathrm{z}$-axis (with associated partial volume averaging effects).

Finally, correlation of the observed MRS metabolite ratio changes with either PET or follow-up MR imaging scans would be paramount to affirm our preliminary findings. While decreased tCho/NAA ratios posttreatment suggest an antiproliferative effect, this inference cannot be determined unequivocally. However, these 18 patients are part of an on- going phase II study at our institution that includes a larger patient sample size, PET scans, and continued interval MR imaging follow-up, designed to assess PFS and overall survival rates in patients receiving IA bevacizumab therapy. Histologic confirmation would have been a powerful adjunct in validating our findings; however, tissue sampling was not performed, given the observed overall favorable radiographic treatment responses.

\section{Conclusions}

This study has demonstrated the promise of MRS as a useful neuroimaging tool in evaluation of tumor extent and therapeutic response in patients treated with bevacizumab. Although the relatively small sample size limits generalization and the aforementioned limitations of anatomic resolution, coregistration errors, and in-plane resolution warrant consideration, the presented results suggest that treatment of recurrent GB with novel SIACI of bevacizumab may be associated with a direct tumoricidal effect, as demonstrated by significant reductions of tCho/NAA ratios in enhancing components of disease after the intervention. Similarly, the trend-level significant reductions of tCho/NAA ratios in nonenhancing T2hyperintense areas of disease suggest a trend toward significance and are promising.

Disclosures: Paul Christos_RELATED: Fees for Participation in Review Activities Such As Data Monitoring Boards, Statistical Analysis, Endpoint Committees: Biostatistical Consulting Core at Weill-Cornell, Comments: Statistical analysis. Apostolos Tsiouris-UNRELATED: Consultancy: BioClinica, Comments: Consult as an independent expert reader for clinical trials; Expert Testimony: Various law firms, Comments: Perform occasional review of legal cases to assess whether standard of care was met; Royalties: Amirsys.

\section{References}

1. Stupp R. Hegi ME, Mason WP, et al. Effects of radiotherapy with concomitant and adjuvant temozolomide versus radiotherapy alone on survival in glioblastoma in a randomised phase III study: 5-year analysis of the EORTCNCIC trial. Lancet Oncol 2009;10:459-66

2. Abraham J. Bevacizumab in recurrent glioblastoma. Commun Oncol 2009;6: 397-400

3. Cohen MH, Shen YL, Keegan P, et al. FDA drug approval summary: bevacizumab (Avastin) as treatment of recurrent glioblastoma multiforme. Oncologist 2009;14:1131-38

4. Mukherji SK. Bevacizumab (Avastin). AJNR Am J Neuroradiol 2010;31:235-36

5. Boockvar JA, Tsiouris AJ, Hofstetter CP, et al. Safety and maximum tolerated dose of superselective intraarterial cerebral infusion of bevacizumab after osmotic blood-brain barrier disruption for recurrent malignant glioma. J Neurosurg 2011;114:624-32

6. Riina HA, Fraser JF, Fralin S, et al. Superselective intraarterial cerebral infusion of bevacizumab: a revival of interventional neuro-oncology for malignant glioma. J Exp Ther Oncol 2009;8:145-50

7. Burkhardt J-K, Riina HA, Shin BJ, et al. Intra-arterial delivery of bevacizumab after blood brain barrier disruption for the treatment of recurrent glioblastoma: progression free survival and overall survival. World Neurosurg 2012;77:130-34

8. Fischer I, Cunliffe $\mathrm{CH}$, Bollo RJ, et al. High-grade glioma before and after treatment with radiation and Avastin: initial observations. J Neurooncol 2008;10:700-08

9. Pope WB, Lai A, Nghiemphu P, et al. MRI in patients with high-grade gliomas treated with bevacizumab and chemotherapy. Neurology 2006;66:1258-60

10. Wagle N, Nghiemphu L, Lai A, et al. Update and developments in the treatment of glioblastoma multiforme - focus on bevacizumab. Pharmacogenomics Pers Med 2010;3:79-85

11. de Groot JF, Yung WKA. Bevacizumab and irinotecan in the treatment of recurrent malignant gliomas. Cancer J 2008;14:279-85

12. Balmaceda C, Critchell D, Mao X, et al. Multisection 1 H magnetic resonance spectroscopic imaging assessment of glioma response to chemotherapy. J Neurooncol 2006;76:185-91

13. Smith EA, Carlos RC, Junck LR, et al. Developing a clinical decision model: MR spectroscopy to differentiate between recurrent tumor and radiation change 
in patients with new contrast-enhancing lesions. AJR Am J Roentgenol 2009;192:W45-52

14. Weybright P, Sundgren PC, Maly P, et al. Differentiation between brain tumor recurrence and radiation injury using MR spectroscopy. AJR Am J Roentgenol 2005;185:1471-76

15. Srinivasan R, Phillips JJ, VandenBerg SR, et al. Ex vivo MR spectroscopic measure differentiates tumor from treatment effects in GBM. Neurooncol 2010;12:1152-61

16. Fan, G. Comments and controversites: magnetic resonance spectroscopy and gliomas. Cancer Imaging 2006;6:113-15

17. Wen PY, MacDonald DR, Reardon DA, et al. Updated response assessment criteria for high-grade gliomas: Response Assessment in Neuro-Oncology Working Group. J ClinOncol 28:1963-72

18. Duyn JH, Gillen J, Sobering G, et al. Multisection proton MR spectroscopic imaging of the brain. Radiology 1993;188:277-82

19. Al-Okaili RN, Krejza J, Wang S, et al. Advanced MR imaging techniques in the diagnosis of intraaxial brain tumors in adults. Radiographics 2006;26:S173-89

20. Lin A, Bluml S, Mamelak AN. Efficacy of proton magnetic resonance spectroscopy in clinical decision making for patients with suspected malignant brain tumors. J Neurooncol 1999;45:69-81

21. Lin A, Ross BD, Harris K, et al. Efficacy of proton magnetic resonance spec- troscopy in neurological diagnosis and neurotherapeutic decision making. NeuroRx 2005;2:197-214

22. McKnight TR, con dem Bussche MH, Vigneron DB, et al. Histopathologica validation of a three-dimensional magnetic resonance spectroscopy index as a predictor of tumor presence. J Neurosurg 2002;97:794-802

23. Hygino da Cruz LC Jr, Rodriguez I, Domingues RC, et al. Pseudoprogression and pseudoreponse: imaging challenges in the assessment of posttreatment glioma. AJNR Am J Neuroradiol 2011;32:1978-85

24. Khasraw M, Lassman AB. Advances in the treatment of malignant gliomas. Curr Oncol Rep 2010;12:26-33

25. Macdonald DR, Cascino TL, Schold SC Jr, et al. Response criteria for phase II studies of supratentorial malignant glioma. J Clin Oncol 1990;8:1277-80

26. Friedman HS, Prados MD, Wen PY, et al. Bevacizumab alone and in combination with irinotecan in recurrent glioblastoma. J Clin Oncol 2009;227:4733-40

27. Dhermain FG, Hau P, Lanferman H, et al. Advanced MRI and PET imaging for assessment of treatment response in patients with gliomas. Lancet Neurol 2010;9:906-20

28. Gerstner ER, Sorensen AG, Jain RK, et al. Advances in neuroimaging techniques for the evaluation of tumor growth, vascular permeability, and angiogenesis in gliomas. Curr Opin Neurol 2008;21:728-35 